\title{
CT ANATOMY OF BUCCAL FAT PAD AND ITS ROLE IN VOLUMETRIC ALTERATIONS OF FACE
}

\author{
R. A. Guryanov ${ }^{\text {a }}$ A. S. Guryanov ${ }^{\text {b }}$ \\ ${ }^{a}$ Sechenov's First Moscow State Medical University, Moscow, Russia - robert.gurianov@gmail.com \\ ${ }^{\mathrm{b}}$ MEDLAZ Clinic, Moscow, Russia - a_gurianov@mail.ru
}

Commission V, WG V/5

KEY WORDS: Buccal Fat Pad Anatomy, Photogrammetry, Computed Tomography, Medical Visualisation

\begin{abstract}
:
The aim of our study is the revision of the anatomy of buccal fat pad and its role in a volumetric pattern of face. Bichat fat pad is a fatty anatomical structure with body and numerous process enclosed between the bony and muscular structures in temporal, pterygopalatine fossae and extents to the cheek area. Nevertheless, the opinion about its structure and role in forming of volume pattern of face sometimes could be controversial. The Bichat fat pad consists on predominately hormone insensitive fat tissue with underdeveloped stroma, this leads to the stability of the fat pad volume and lesser radiodensity in contrast to the subcutaneous fat. Moreover, the buccal fat pad is delimited from the subcutaneous fat of cheek area by the strong capsule. This feature allows us to use CT to divide the Bichat fat pad from the surrounding tissues. The thorough embryological data provide the distinction of Bichat fat pad from the subcutaneous fat of cheek area even at the stage of development. On the other hand, the border between the masticatory muscles and the processes of the fat pad is not evident and resembles cellular spaces in the other anatomical areas. To elicit the role of the buccal fat pad in volume pattern of face and its function we have performed the several experiments, analyzed the postoperative results after Bichat fat pad resection using surface scanner and CT data. At first, we have performed the gravity test: the patient's face photogrammetry scanning in horizontal and vertical position of head and it revealed the excess of volume in temporal area in horizontal position. To exclude mechanism of overflowing of the skin and subcutaneous fat over the zygomatic arch we have placed the markers on the skin surface at the different areas of face including the projection of ligaments and found out that the migration of soft tissue over the zygomatic arch is about $3-5 \mathrm{~mm}$ and almost the same in temporal area. However, the acquired result was unsatisfying because cannot exclude completely the migration of superficial tissues. In following experiments it was shown that the intensive pressure on the cheek area in vertical position produce the volume excess in the temporal area similar and more exaggerate than in gravity test. To correlate the excess of tissue with underlying anatomical structures we had acquired the CT's of some probationers, performed 3D reconstruction of bony structures, Bichat fat pad, and aligned with the previous surface scans. The projection of this excess in both experiments corresponds with the temporal process of Bichat fat pad. That means that the leading mechanism of these changes is protrusion of temporal process of Bichat fat pad through the leaves of temporal fascia due to pressure on the buccal extension: in these conditions, the buccal fat pad works as a communicating vessel between the cheek area and temporal fossa. This fact has suggested us that the phenomenon of the deepening of temporal area during the ageing could be produced as by the atrophy of buccal fat pad as by the migration of the fat pad to the cheek area due to ptosis.
\end{abstract}

\section{INTRODUCTION}

The volumetric appearance of the human face is initially defined by the bony structures that serve as the rigid frame for the soft tissues. The soft tissues of the face is a complex system of subcutaneous fat, SMAS, mimic muscles ligaments also includes the deep fat compartment of face is known as the buccal [Bichat] fat pad. The main feature of the soft tissues is their higher inconstancy, mobility in comparison with bones so the former are vastly subjected to adaptive and age related changes. Bachat fat pad (BFP) consists of a main part - the body, and several extensions arising from it: masseteric extension, preigomandibular extension, orbital extension, pterygopalatine extension, temporal extension (Kahn, J.L., Wolfram-Gabel, R. \& Bourjat, P., 2000, Yousuf, S. et al., 2010). The extension`s anatomy is spatially complex, because they fill the space between the masticatory muscles $(\mathrm{m}$. masseter, m. pterygoideus medialis et lateralis, m. temporalis). Nowadays, the distinct physiological role of Bichat fat pad is not clearly defined, surgeons consider the fat pad as the source of plastic material and as a body-to-resect in cases when patient unsatisfied by the cheek plumpness, also the buccal fat pad regarded by some surgeons as the morphological substance for jowls formation (Zhang, H.-M. et al., 2002, Yousuf, S. et al., 2010). Nevertheless, the volumetric role of Bichat fat pad is significant and takes sufficient part in forming the midface appearance. The aim of our work is to revise the anatomy of Bichat fat pad and to clarify its role in the face volumetric appearance using such noninvasive visualization technics as the CT reconstruction and photogrammetry.

\section{MATERIALS AND METHODS}

\subsection{Photogrammetry study}

Photogrammetry allows us to analyze the changes in volumetric pattern of face under the variety of factors as the gravitation or premediated mechanical impact. This factors open up the possibility to understand the general regularities of the soft tissue migration and correlate our findings with the anatomical and clinical observations. 


\subsubsection{Gravity test}

The main force that effects constantly on the soft tissues of face is gravity. We have developed the test that could cast the light on the mechanisms of aging and ptosis, and reveals the dynamic of volumes migration, the zones of adhesion of soft tissues to the bony framework. We have called that test the gravity test. Using photogrammetry the scanning of probationers face is performed in vertical and horizontal positions. The acquired scans are aligned regarding the reference points - the points or surfaces in which volume changing and migration of tissues is predictably insignificant due to reliable fixation to the bones.

Then on the aligned models, we compute the geodesic map (distance map) of difference between shapes of face in vertical in horizontal position, so the migration of volume reveals. We have analyzed the pattern of volumetric changes in 20 of different age groups, including 7 congener pairs (motherdaughter). In addition, we perform the modification of test (5 cases) in which the marks are placed on the surface of skin, thus the migration of skin surface can be observed. This modification of gravity test allows us to estimate the mobility of skin surface regarding the bony structures, hence in immovable areas correlate with ligaments between the derma and bones and the migration of soft tissues under the force of gravitation in that area is confined. In the point of maximal protrusion, we calculate the distance between the aligned models what permits us to access the grade of protrusion. In view of that the elasticity of skin is able to effect on the grade of protrusion we have used the indirect parameter - relative extension coefficient to estimate he elasticity of the skin.

\subsubsection{Cheek-pressing test}

The temporal fossa and the cheek area communicates with the cheek area through the masticatory space enclosed between the masseter laterally. Cheek pressing test is performed in vertical position, the probationer should push the cheek in projection of buccal extension of Bichat fat pad by his own finger, and the photogrammetry scans are taking at this moment. The scan is aligned with the scan in vertical position and the geodesic map is computing. The acquired geodesic map is compared with that of gravity test. This test imitates the mechanism of soft tissues' pressure on the buccal fat pad.

\subsubsection{Clench test}

When we clench our teeth, the masticatory muscles increase their volume due to contraction and gain of blood inflow. The similar effect occurs during the act of mastication. Thus, the clench test shows how the muscles effect on the volumetric pattern of human face, and how the mastication effect on the Bichat fat pad volume migration.

\subsection{CT reconstruction}

The method of 3D reconstruction has been used to access the projection of the buccal fat pad on the surface of face and to elicit the topography of fat pad. The separation of Bichat fat pad in can arouse some complexities: first, we have to mind that in a cheek area the buccal fat pad has a strong capsule that delimit the fat pad from the subcutaneous fat; second, in the temporal area the fat pad should be distinguished from the interfascial fat between the two leaves of temporal fascia (Cho, K.H. et al., 2013). The fascia's and BFC (-69 to $-49 \mathrm{HU})$ is much dense than a fatty tissue consisting predominately of adipocytes (-179 to $-124 \mathrm{HU}$ ) in radiodensity scale. This fact allows us to perform the virtual dissection of the buccal fat pad. We had analysed the series of 10 patient's CTs of head, including two patients after BFP resection. The examination of BFP has being performed on the multiplane and 3D reconstructions: the capsule of the Bichat fat pad, topographic anatomy features, position of processes were accessed.

\section{RESULTS}

The gravity test has shown the significant increase of volume in the temporal area. The mean value of increase was $2.35 \mathrm{~mm}$, and can be observed visually. The specific pattern of volumetric changes in the temporal area has been observed (fig.1). The area with volume excess has the evident limit below over the zygomatic arch. The footprint of the protrusion varies in individuals, but never fill the whole temporal fossa and never appears at the areas where we can confidently palpate the temporalis muscle. This phenomenon has stimulate us to find the anatomical base for it. We have performed the CTs of our patients and have revised the anatomy of buccal fat pad on multiplane reconstruction and 3D reconstruction. In the cheek area the buccal fat pad has a considerable capsule, which allows us to divide it from the subcutaneous fat. On the other hand, in temporal area there is no distinct border between the masticatory muscles and BFP it and the latter is directly adjacent to the former with no distinguishable capsule, but laterally it is strongly delineated by the deep leaf of temporal fascia from interfascial fat compartment enclosed between the superficial and profound leaves of temporal fascia.

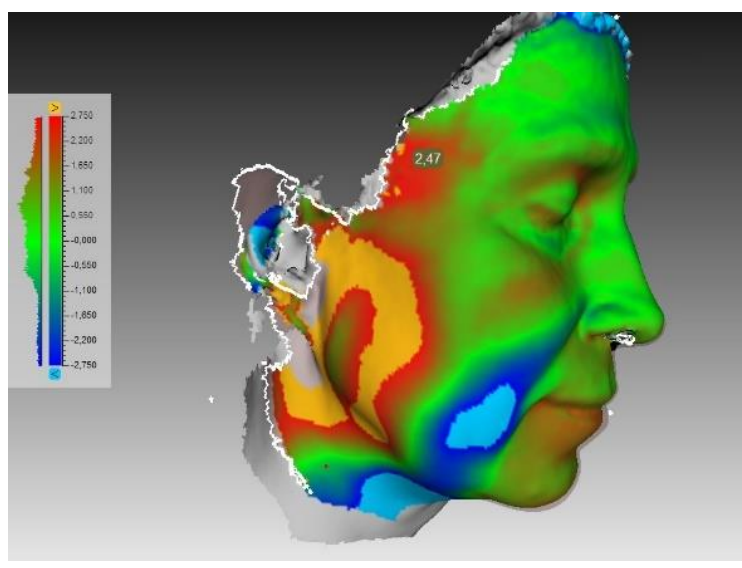

Figure 1 . The gravity test. Geodesic measurements. Green - no volume changes. Blue - diminishing of volume. Red augmentation of volume. The isolated excess of volume in temporal area marked with a surface distance value.

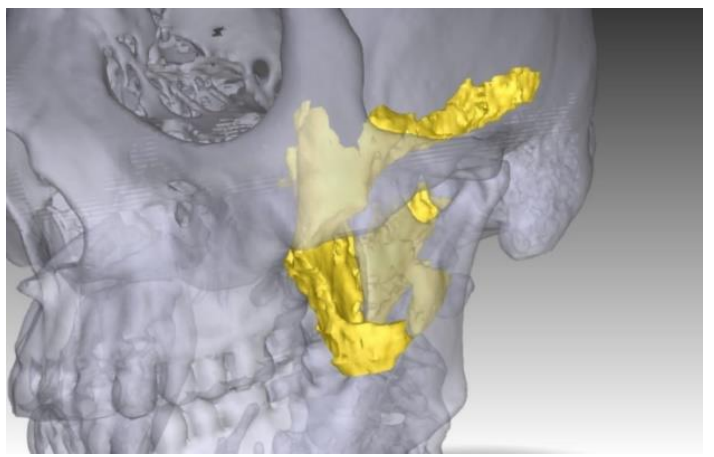

Figure 2. Reconstruction of the buccal fat pad. The connection between the buccal process and temporalis process is shown. 
3D reconstruction of BFP allowed us to estimate the spatial orientation of the processes and understand how the BFP projects on the skin surface. The buccal process is connected with the temporal process via the body of buccal fat pad. (fig. 2)The area of protrusion in the temporal area corresponds with the projection of temporal process of BFP on the skin surface (fig.3). This permits us to conclude that the protrusion in the temporal area at gravity test appears due to the migration of Bichat fat pad and the pressure of overlying midface tissues on it. Nevertheless, we presumed that the increase of volume in temporal area can be conditioned by two mechanisms the migration of the tissues over or under the zygomatic arch.

To exclude mechanism of overflowing of the skin and subcutaneous fat over the zygomatic arch we have placed the markers on the skin surface at the different areas of face including the projection of ligaments and found out that the migration of soft tissue over the zygomatic arch an in projection of malar bone is about 3-5 $\mathrm{mm}$ and almost the same in temporal area (fig. 4). However, the acquired result was unsatisfying because cannot exclude completely the migration of superficial tissues (skin, subcutaneous fat), but it strongly demonstrate us that the migration over that structures is limited as the volumetric changes.

At the gravity test in the congener pairs, it has become obvious that the area of protrusion in temporal area definitely expands in age. This can occur due to the diminishing of elasticity of the soft tissue during the ageing.

The cheek-pressing test revealed that the pressing in a projection of body of the Bichat fat pad can produce the protrusion in the temporal area (fig. 5), less prominent though (approximately 58\% of temporal excess in gravity test). This fact confirm our supposition that the pressure applied to the buccal part of the BFP's body is able to cause the protrusion in the temporal area.

At the clench test the protrusion also appears in the temporal area, but also the increase of volume is observed at the beginning of temporalis muscle, where the buccal fat pad is absent, and at the contour of masseter muscle. We suppose that the part of the protrusion just above the zygomatic arch concerns to the buccal fat pad.

\section{DISCUSSION}

This study is trying to explain the volumetric alteration of face under the action of different forces. It is known that the atrophy of buccal fat pads develops during the aging, but being hormone insensitive the Bichat fat pad volume do not depends of grade of obesity, thus even on the cachectic patients it saves its volume constant, in contrast to subcutaneous fat (Loukas, M. et al., 2006). In embryogenesis, the BFP also develops separately from the other fatty structures: the capsule of BFP forms first and only after that, the differentiation of the fatty tissue begins (Cho, K.H. et al., 2013). This suggests that the BFP has its own individual function (because the function is always connected with morphology) which is not clearly defined nowadays (Yousuf, S. et al., 2010).

It is noticeable that the excess of volume in temporal area at the accomplished experiments has a mediated mechanism. At first the force of gravity acts on the soft tissues, which effects on the buccal process and the body of Bichat fat pad. Bichat fat pad conducts the pressure to the deep leaf of temporal fascia, interfascial fat, superior leaf of temporal fascia and, finally, the skin. The fatty tissue of BFP, having lack of stroma, consisting of lipids and being incompressible, playing the role of volumetric buffer of temporal, infratemporal areas, thus the buccal fat pad works as a communicating vessel between the cheek area and temporal fossa. That means that at the act of mastication the muscles would not be restrained in their compartments during the chewing. The thick fascial leaves of temporalis fascia limits the temporal fossa, so the temporalis muscle is enclosed in a kind of hard compartment, limited laterally by the temporalis fascia, medially by the synergic pterygoid muscles. With no presence of BFP the masticatory muscles due to ingrowing inflow and function would be squeezed in their receptacles arousing pain and subsequent inability to chew.

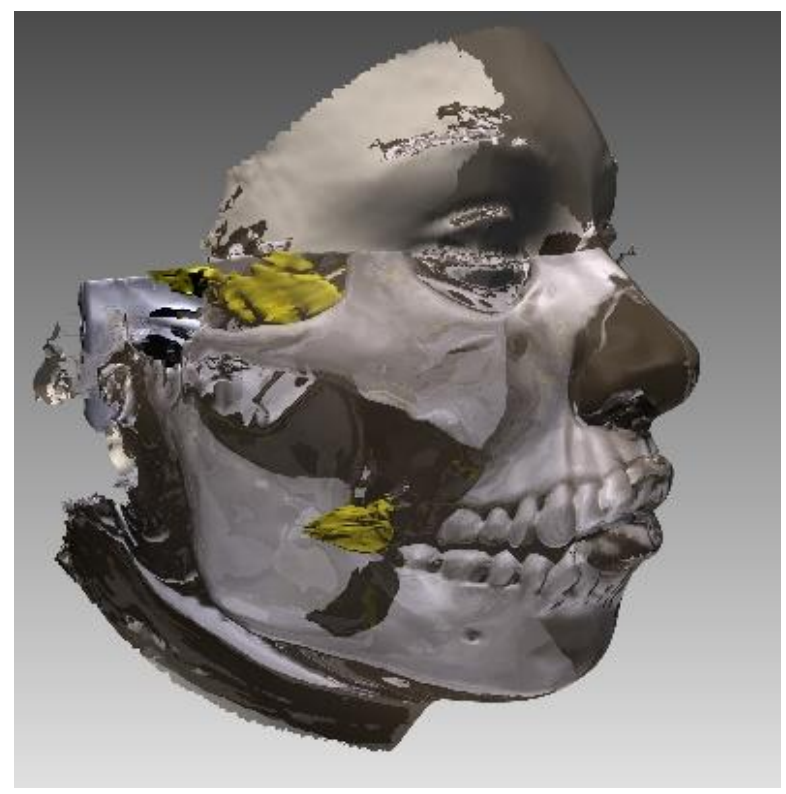

Figure 3. The temporal process of buccal fat pad: temporal and buccal processes.

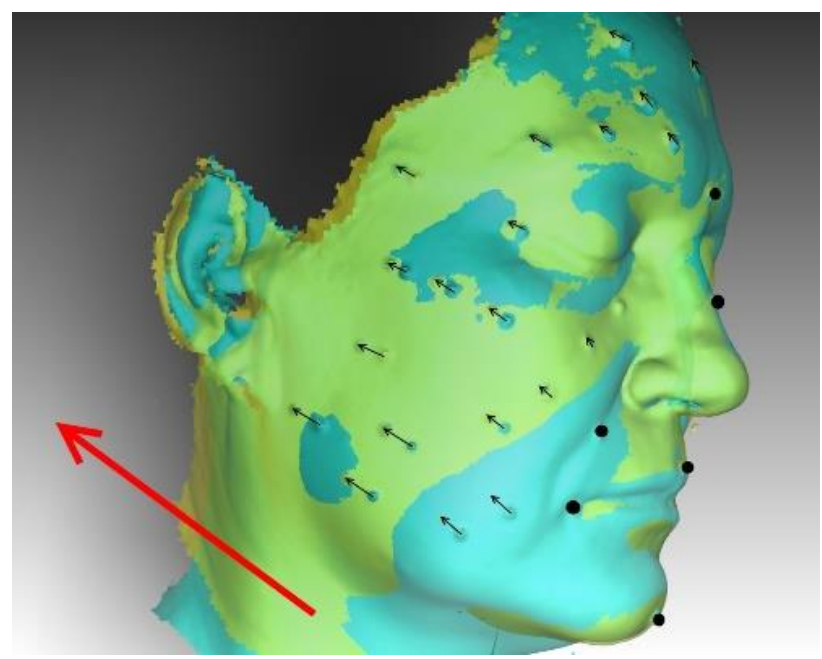

Figure 4. The variation of gravity test with marks. The little migration over the zygomatic arch is present. 


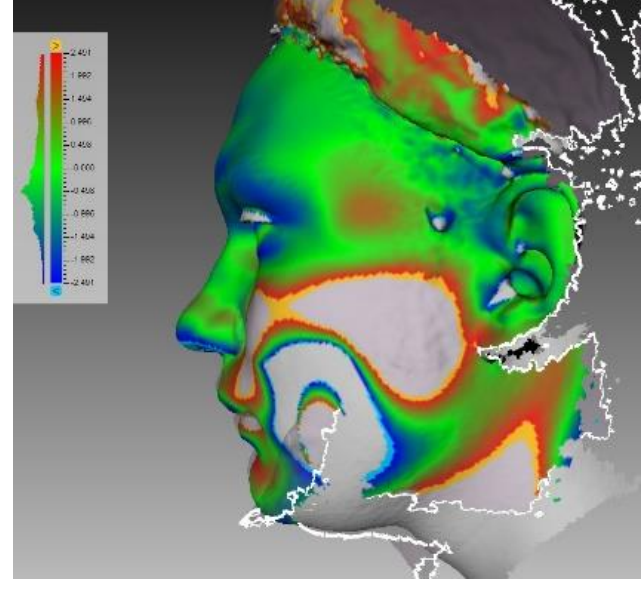

Figure 5. Pressing test. The protrusion over the zygomatic arch.

Computed tomography reconstructions demonstrates the connection between the temporal and cheek areas by the BFP. The temporal, infraorbital, pterygopalatine processes of buccal fat pad connect the deep areas of face with the superficially lying cheek area, so the septic process can spread along this fatty tract and involve the structures as the nerves, venous plexuses, arteries and can spread on the other adjacent structures.

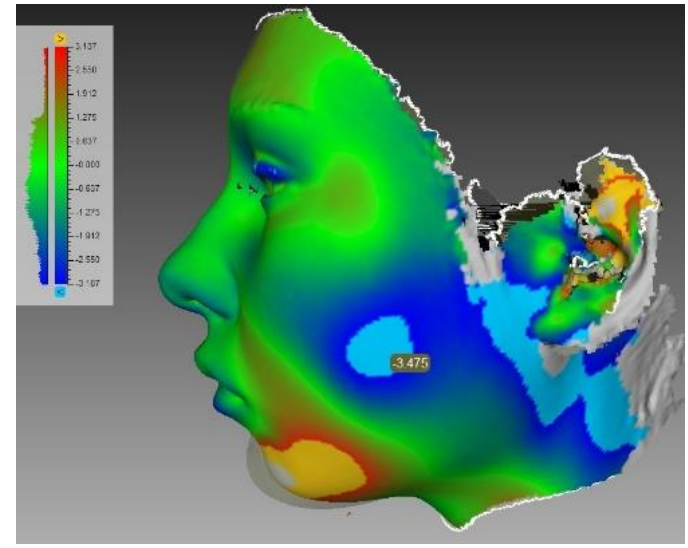

Figure 6. Geodesic map after the buccal fat pad resection and augmentation mentoplasty. The decrease of volume in the check area in front of anterior margin of masseter muscle.

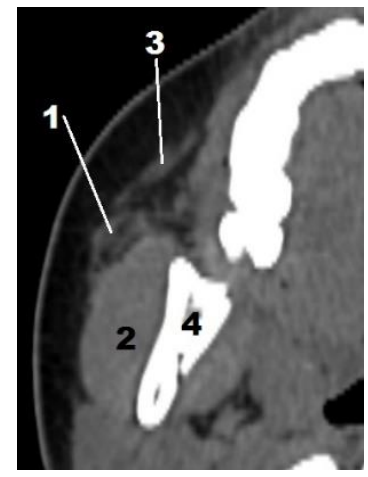

Figure 7. BFP after resection. 1 - fibrosis in the projection of BFP; 2 - masseter muscle; 3 -parotid duct; 4 - ramus mandibuli
From the view of plastic surgery, the Bichat fat pad and its size plays the significant role in the appearance of face. The resection of fat pad decrease the volume in the specific area at the front margin of the masseter muscle, but surprisingly not affecting the temporal area (fig. 6). The only fact that can explain this is that on the CT's the strong fibrosis (fig.7) of the resected buccal part was observed, so it hinders the temporal and the latter parts to prolapse into the freed space. On the other hand, we know that the Bichat fat pad has a developed capsule only in the cheek area, the part that is enclosed between the muscles has loose septs connected directly to the epimiseum of masticatory muscles (Kahn, J.L., Wolfram-Gabel, R. \& Bourjat, P., 2000), and they could prevent the alteration in the volumetric appearance of the temporal area.

The ptosis of the soft tissues appears due to continuous action of gravitation during the aging. Decreasing elasticity of the connective tissue cannot prevent the migration of the soft tissues downwards. Many reports that the volume of the buccal fat pad and another fatty tissue of face decreases in age (Gierloff, M. et al., 2012, Loukas, M. et al., 2006, Xiao, H., M. Bayramiçli, I.T. Jackson, 1999), but not takes into account that the enhancement migration ability may be caused by the decreasing elasticity of the soft tissue compartment, including the compartment of the whole body - the skin. The expanding of protrusion area in age may be connected with decreased elasticity of connective tissue strictures, thus the deepening in the temporal area has the mechanism inverse to that in gravity test.

\section{REFERENCES}

Cho, K.H. et al., 2013. Deep fat of the face revisited. Clinical Anatomy, 26(3), pp.347-356.

Gierloff, M. et al., 2012. Aging Changes of the Midfacial Fat Compartments. Plastic and Reconstructive Surgery, 129(1), pp.263-273.

Kahn, J.L., Wolfram-Gabel, R. \& Bourjat, P., 2000. Anatomy and imaging of the deep fat of the face. Clinical Anatomy, 13(5), pp.373-382.

Loukas, M. et al., 2006. Gross anatomical, CT and MRI analyses of the buccal fat pad with special emphasis on volumetric variations. Surgical and Radiologic Anatomy, 28(3), pp.254-260.

Xiao, H., M. Bayramiçli, I.T. Jackson, 1999. Volumetric analysis of the buccal fat pad Eur J Plas Surg 22:177-181

Yousuf, S. et al., 2010. A review of the gross anatomy, functions, pathology, and clinical uses of the buccal fat pad. Surgical and Radiologic Anatomy, 32(5), pp.427-436.

Zhang, H.-M. et al., 2002. Anatomical structure of the buccal fat pad and its clinical adaptations. Plastic and reconstructive surgery, 109(7), pp.2509-2518; discussion 2519-2520. 